\title{
Sensory information in local field potentials and spikes from visual and auditory cortices: time scales and frequency bands
}

\author{
Andrei Belitski • Stefano Panzeri • Cesare Magri • \\ Nikos K. Logothetis • Christoph Kayser
}

Received: 14 August 2009 / Revised: 17 February 2010 / Accepted: 26 February 2010 / Published online: 16 March 2010

(C) The Author(s) 2010. This article is published with open access at Springerlink.com

\begin{abstract}
Studies analyzing sensory cortical processing or trying to decode brain activity often rely on a combination of different electrophysiological signals, such as local field potentials (LFPs) and spiking activity. Understanding the relation between these signals and sensory stimuli and between different components of these signals is hence of great interest. We here provide an analysis of LFPs and spiking activity recorded from visual and auditory cortex during stimulation with natural stimuli. In particular, we focus on the time scales on which different components of these signals are informative about the stimulus, and on the dependencies between different components of these signals. Addressing the first question, we find that stimulus information in low frequency bands $(<12 \mathrm{~Hz})$ is high, regardless of whether their energy is computed at the scale of milliseconds or seconds. Stimulus information in higher bands $(>50 \mathrm{~Hz})$, in contrast, is scale dependent, and is
\end{abstract}

\section{Action Editor: Gaute T Einevoll}

Electronic supplementary material The online version of this article (doi:10.1007/s10827-010-0230-y) contains supplementary material, which is available to authorized users.

A. Belitski • N. K. Logothetis $\cdot$ C. Kayser $(\triangle)$

Max Planck Institute for Biological Cybernetics,

Spemannstrasse 38,

72076 Tübingen, Germany

e-mail: christoph.kayser@tuebingen.mpg.de

S. Panzeri $(\bowtie) \cdot$ C. Magri

Robotics, Brain and Cognitive Sciences Department,

Italian Institute of Technology,

Via Morego 30, 16163 Genoa, Italy

e-mail: stefano.panzeri@iit.it

N. K. Logothetis

Division of Imaging Science and Biomedical Engineering,

University of Manchester,

Manchester M13 9PT, UK larger when the energy is averaged over several hundreds of milliseconds. Indeed, combined analysis of signal reliability and information revealed that the energy of slow LFP fluctuations is well related to the stimulus even when considering individual or few cycles, while the energy of fast LFP oscillations carries information only when averaged over many cycles. Addressing the second question, we find that stimulus information in different LFP bands, and in different LFP bands and spiking activity, is largely independent regardless of time scale or sensory system. Taken together, these findings suggest that different LFP bands represent dynamic natural stimuli on distinct time scales and together provide a potentially rich source of information for sensory processing or decoding brain activity.

Keywords Information theory - Vision - Audition · Population coding $\cdot$ Oscillations $\cdot$ Firing rates

\section{Introduction}

The neural signals commonly measured with microelectrodes consist of action potentials and slow-wave activity known as local field potentials. Spikes can be detected and classified by analyzing the high-frequency range (typically $>5000 \mathrm{~Hz}$ ) of the extracellular signal, whereas the local field potential (LFP) is assessed by the power variation in the low-frequency range (typically below $100 \mathrm{~Hz}$ ). The LFP is sensitive to subthreshold integrative processes and carries information about the state of the cortical network, which is difficult to capture using spiking activity from only a few neurons (Buzsaki 2006; Logothetis 2002; Mazzoni et al. 2008; Mitzdorf 1985). Field potentials include population synaptic potentials (Juergens et al. 1999; Mitzdorf 1985) and various types of slow activity 
such as voltage-dependent membrane oscillations and spike afterpotentials (Buzsaki 2002; Buzsaki et al. 1988; Logothetis 2008; Ray et al. 2008a). LFPs are also thought to reflect the degree of balance between excitation and inhibition and the degree of engagement of excitatoryinhibitory interactions (Bartos et al. 2007; Brunel and Wang 2003; Cardin et al. 2009; Logothetis 2008; Mazzoni et al. 2008). Although earlier studies on sensory function focused mostly on spiking activity, more recent studies highlighted the importance of considering the stimulus induced changes in field potentials (Belitski et al. 2008; Berens et al. 2008; Frien et al. 2000; Kayser and Konig 2004; Liu and Newsome 2006; Pettersen and Einevoll 2008; Siegel and Konig 2003). Since LFPs are sensitive to supra and subthreshold processes, investigating stimulus encoding by LFPs can offer additional insights into sensory representations beyond those offered by only measuring neuronal spike trains (Logothetis 2002; Nicolelis and Lebedev 2009; Ray et al. 2008b).

Dynamic naturalistic stimuli evolve on multiple temporal scales and are characterized by complex dynamics (Dong and Atick 1995; Kayser et al. 2003a; Singh and Theunissen 2003). This begs the question of what time scales in neural signals such as LFPs are related to the sensory stimulus and provide information about this. In a previous study (Belitski et al. 2008) we made a first step in this direction and considered how LFPs and spikes recorded from visual cortex encode information about slow changes in natural movies occurring on the scale of seconds. In the present study we address this question more systematically and analyze LFPs and spiking activity (MUA) recorded from visual and auditory cortices during stimulation with natural stimuli. In particular, we focus on two questions: what are the time scales on which different components (frequency bands) of the LFP are informative about the stimulus, and what are the dependencies between different LFP bands and between LFP bands and MUA in representing sensory stimuli.

\section{Materials and methods}

We analyzed sensory evoked LFPs and MUA recorded from primary visual cortex (V1) of anaesthetized monkeys and caudal auditory cortex of alert monkeys. The recording procedures and stimulation paradigms for the different datasets are described shortly in the following and have been detailed previously (Belitski et al. 2008; Kayser et al. 2009; Montemurro et al. 2008). All experiments were approved by the local authorities (Regierungspräsidium) and were in full compliance with the guidelines of the European Community (EUVD 86/609/EEC) for the care and use of laboratory animals.

\subsection{Recordings in primary visual cortex}

Recordings (23 sites) from V1 of two adult rhesus monkeys (Macaca mulatta) were obtained while the animals were anaesthetized (remifentanyl, $1 \mu \mathrm{g} / \mathrm{kg} / \mathrm{min}$ ), muscle-relaxed (mivacurium, $5 \mathrm{mg} / \mathrm{kg} / \mathrm{h}$ ) and ventilated (end-tidal $\mathrm{CO}_{2}$ $33 \mathrm{mmHg}$, oxygen saturation $>95 \%$ ). Body temperature was kept constant and lactated Ringer's solution supplied $(10 \mathrm{ml} / \mathrm{kg} / \mathrm{h})$. Vital signs (SpO2, ECG, blood pressure, endtidal $\mathrm{CO}_{2}$ ) were continuously monitored. Neuronal activity was recorded from opercular V1 (foval and para-foveal representations) using microelectrodes (FHC Inc., Bowdoinham, Maine, 300-800k Ohms), signals were high-pass filtered ( $1 \mathrm{~Hz}$, digital two pole Butterworth filter), amplified using an Alpha Omega amplifier system (Alpha Omega Engineering) and digitized at $20.83 \mathrm{kHz}$. Binocular visual stimuli were presented at a resolution of $640 \times 480$ pixels (field of view: $30 \times 23$ degrees, 24 bit true color, $60 \mathrm{~Hz}$ refresh) using a fiberoptic system (Avotec, Silent Vision, Florida). Stimuli consisted of 'naturalistic' complex and commercially available movies $(30 \mathrm{~Hz}$ frame rate), from which $3.5-6$ min long sequences were presented and repeated 30-40 times (Star Wars Episode 4 and The Last Samurai). We confirmed that the receptive fields of all neurons were within area of visual stimulation (Rasch et al. 2008).

\subsection{Recordings in auditory cortex}

Recordings ( $n=76$ sites) were obtained from three adult rhesus monkeys (Macaca mulatta) passively listening to sounds in an anechoic booth. Microelectrodes (FHC Inc., Bowdoinham, Maine, 0.8 to $6 \mathrm{MOhms}$ ) were spaced at $750 \mu \mathrm{m}$, signals were amplified using a modified Alpha Omega system (Alpha Omega Engineering), filtered between $4 \mathrm{~Hz}$ and $9 \mathrm{kHz}$ (4-point Butterworth filter) and digitized at $20.83 \mathrm{kHz}$. Recording sites covered the auditory core (primary auditory cortex) and caudal belt regions. Sounds (average intensity $65 \mathrm{~dB}$ SPL) were delivered from two free field speakers (JBL Professional) positioned 50 degrees to the left and right at $70 \mathrm{~cm}$ distance from the head. A continuous $52 \mathrm{sec}$ sequence of natural sounds was repeated at least 55 times for each site. This sequence was created by concatenating 21 each 1-4 sec long snippets of various naturalistic sounds (including animal vocalizations, environmental sounds and conspecific macaque vocalizations).

\subsection{Signal extraction}

Both datasets were processed using the same resampling and filtering procedures. In detail, the LFP was extracted by band-pass filtering the neural signal and resampling at a rate of $250 \mathrm{~Hz}$. Filtering was done using Kaiser filters with sharp transition bandwidth $(1 \mathrm{~Hz})$, small passband ripple 
$(0.01 \mathrm{db})$ and high stopband attenuation $(60 \mathrm{~dB})$. A mirroring technique was used to reduce edge artifacts during filtering and forwards and backwards filtering was used to eliminate phase shifts. Individual frequency bands were spaced at $4 \mathrm{~Hz}$ between 1-100 Hz, with the exact bands being 4-8 Hz, 8$12 \mathrm{~Hz}, \ldots, 96-100 \mathrm{~Hz}$. For each band we first computed the Hilbert transform and from this the instantaneous energy as the square of the modulus of the transform (Fig. 1). The amplitude of the Hilbert transform is also known as the band's envelope. Multi-unit spiking activity was extracted by high-pass filtering the raw signal above $500 \mathrm{~Hz}$ and extracting spike times by threshold crossing (with threshold set to 3.5 SD) for the visual cortex data (see (Belitski et al. 2008) for full details) and using commercial spike-sorting software (Plexon Offline Sorter) for the auditory data (see (Kayser et al. 2009) for full details). For the present analysis we did not separate single and multi-units.

\subsection{Information theoretic analysis}

and stimulus characterization

To determine whether the energy of a given LFP band is related to the sensory stimulus we computed the mutual Shannon information between stimulus and response (Shannon 1948), which is defined as

$I(S ; R)=\sum_{r, s} P(s) P(r \mid s) \log _{2} \frac{P(r \mid s)}{P(r)}$
Here $\mathrm{P}(s)$ is the probability of presenting stimulus $s, \mathrm{P}(r \mid s)$ is the probability of observing the response $r$ given presentation of stimulus $s$, and $\mathrm{P}(r)$ is the probability of observing response $r$ across all trials to any stimulus. $I(S ; R)$ quantifies the reduction of uncertainty about the stimulus that can be gained from observation of a single trial response in units of bits. $I(S ; R)$ is zero only when the stimulus and the response are statistically independent, indicating that no knowledge about the stimulus can be gained by observing the response.

In this study the neural responses $r$ was defined in four different ways, giving rise to slightly different information calculations. In the first case, $r$ was defined as the energy of the LFP in a given frequency band $f$, and the resulting information $\mathrm{I}(\mathrm{S} ; \mathrm{R})$ calculated in this way was denoted as $I\left(S, R_{f}\right)$. In the second case, when computing the information $\mathrm{I}\left(\mathrm{S} ; \mathrm{R}_{\mathrm{f} 1} \mathrm{R}_{\mathrm{f} 2}\right)$ in the joint observation of LFP energy at two different frequencies, $r$ was a twodimensional array $\left(R_{f 1}, R_{f 2}\right)$ containing the energy simultaneously observed at two different frequencies $f_{1}$ and $f_{2}$. In the third case we computed the information carried by the spike count, and $r$ was the number of spikes observed in a trial. In the fourth case, when computing the information in the joint information carried by spike counts and LFP energy at a given frequency, the response $r$ was a two-dimensional array containing the energy of LFP at a given frequency $\mathrm{f}$ in one dimension and the spike count in the other dimension.
Fig. 1 Information theoretic analysis of field potentials (a) For the information analysis the time axis during stimulus (movie or sound) presentation was divided into non-overlapping windows

$\left(\mathrm{S}_{\mathrm{i}}\right)$ of length $T$, within which the responses were characterized. In this formalism, a given response can be elicited by any sensory feature either occurring in the respective time window $i$, or in any previous time window. The window length $\mathrm{T}$ was varied systematically from 4 to 2048 ms. (b) Example data from auditory cortex illustrating the raw LFP from a single trial, together with the band-pass filtered signal in the $4-8 \mathrm{~Hz}$ band. The red line denotes the energy of the signal, extracted by the Hilbert transform. The lower panel displays the binned $(n=4)$ energy in several subsequent trials. Arrows indicate instances where the energy is highly consistent across trials (a) Movie or sound

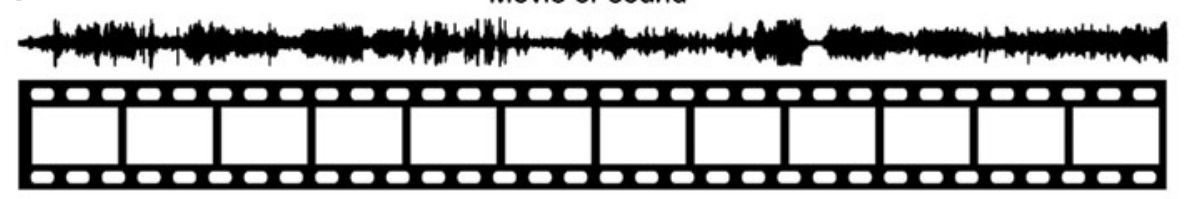

Temporal windows (= stimuli)

(b)
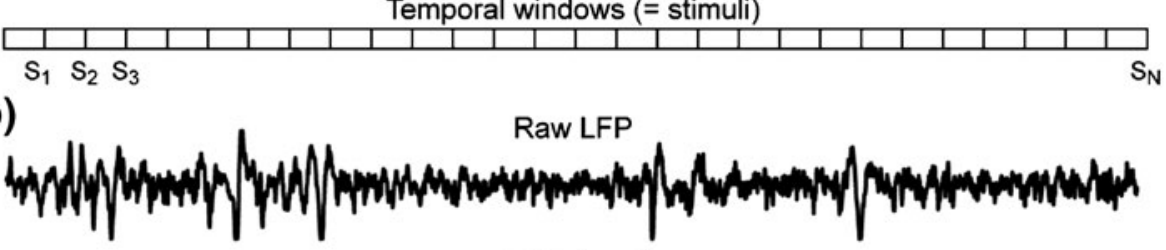

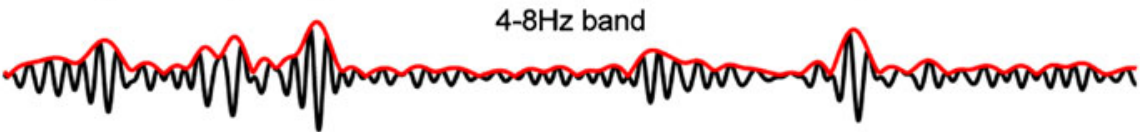

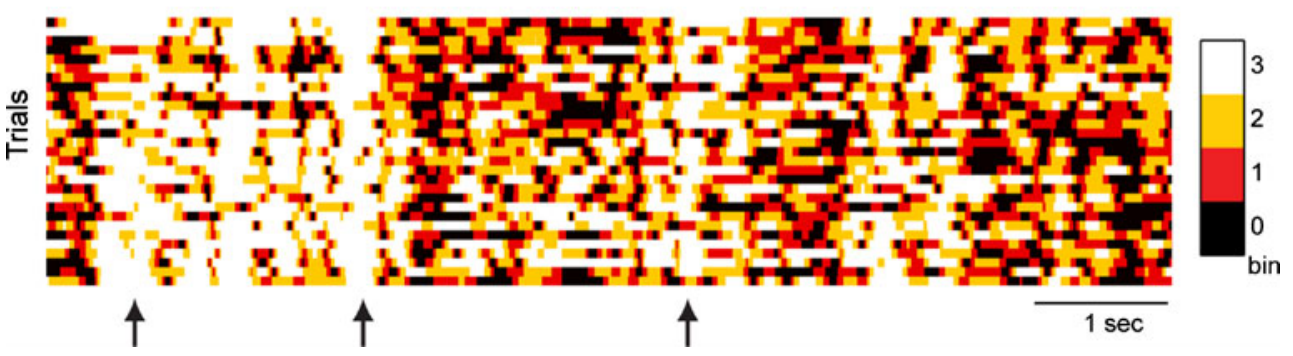


To compute the mutual information between LFP and sensory stimulus $S$, we used a feature independent definition of stimulus, described next (de Ruyter van Steveninck et al. 1997; Strong et al. 1998). The stimulation time was divided into non-overlapping time windows $T$, and the LFP or spiking response was collected separately in each of these windows. Each window was labeled with a stimulus identification number $(\mathrm{s}=1,2 \ldots)$, and we computed the information between the response and the identity of the window in which it was elicited (i.e. the section of the dynamic stimulus that provoked the response). It is important to note that the response collected in the s-th window is potentially elicited by the whole temporal stimulation pattern up to the time point of window s. The stimulation history is (by experimental design) exactly the same from trial to trial. Therefore this definition of stimulus information from the response in a given window takes into account the potential contributions of all stimulus features at all previous times windows in that trial. As such, and although for simplicity we will denote the windows in which the response in collected as "stimulus windows", the information calculation does not assume an instantaneous relationship between LFPs and stimuli, and takes into account the effects of all time lags between neural response and any stimulus feature that provoked it. This definition of stimulus space has the advantage that it does not make any assumptions about which stimulus features or combinations thereof provokes a neuronal response (either presented at the current time or in the period prior to the considered neural response). This also makes the information value formally invariant to any rigid time shift between stimulus windows and neural response corresponding to any assumption of a given latency value between stimuli and responses. A schematic of this procedure is provided in Fig. 1(a). The length of the time window $T$ is a parameter which (as detailed in the Results) was varied parametrically in the range from 4 to $2048 \mathrm{~ms}$, in order to evaluate the temporal precision with which the neural signal can segment the natural dynamic stimulus.

The information synergy between the LFP energies at two different frequencies was defined as the percent difference between the information provided by the joint signals $\left(f_{1}, f_{2}\right)$ and the sum of the independent information values (Belitski et al. 2008; Hatsopoulos et al. 1998): Synergy $=100 *\left[\mathrm{I}\left(\mathrm{S}, \mathrm{f}_{1}, \mathrm{f}_{2}\right)-\left(\mathrm{I}\left(\mathrm{S}, \mathrm{f}_{1}\right)+\mathrm{I}\left(\mathrm{S}, \mathrm{f}_{2}\right)\right)\right] /\left(\mathrm{I}\left(\mathrm{S}, \mathrm{f}_{1}\right)+\mathrm{I}\right.$ $\left.\left(\mathrm{S}, \mathrm{f}_{2}\right)\right)$. Negative values of this index indicate redundancy. The information synergy between the information carried by spike counts and that carried by LFPs energy at a given frequency was defined by a straightforward extension of the above.

The computation of the above information quantities requires the estimation of stimulus-conditional response probabilities $P(r \mid s)$. These probabilities are not known a priori and must be measured experimentally from a finite number of trials. Even when each stimulus is repeated many times, as was the case here, the estimated probabilities suffer from finite sampling errors, which induce a systematic error (bias) in information estimates (Panzeri et al. 2007). We used the following procedure to correct for the bias. To facilitate the sampling of its probability, we discretized the response space by binning the response power into $n=4$ equipopulated bins. We then computed the information by simply "plugging" the empirical binned probabilities into the information equations and corrected for the bias as follows. First, we used a quadratic extrapolation procedure (Strong et al. 1998) to estimate and subtract out the bias of each information quantity. When computing the information conveyed by the joint observation of two responses (such as LFP energies at different frequencies) we also applied the "shuffling procedure" described in (Montemurro et al. 2007; Panzeri et al. 2007), which greatly reduces the bias of multidimensional information estimates and typically makes the residual bias negative. This implies that our estimates of the joint information carried by two signals (and thus of synergy) are slightly biased downward. We then checked for residual bias by a "bootstrap procedure": stimuli and responses were paired at random, and the information for these random pairings was computed. Since in this random case the information should be zero, the resulting value is an indication of a residual error. For all data reported here, the residual information in randomized data was minimal, demonstrating that the statistical biases were well controlled, and it was anyway subtracted out of the final information estimate.

We implemented the information calculations using routines from the Information Breakdown Toolbox (Magri et al. 2009). We tested, by means of simulated LFP power responses with statistics matching that of the LFP responses in the visual dataset (see Supplemental Material, section "Simulated LFP data"), and we found that the estimates of both single and double frequency mutual information I(S,f) and $\mathrm{I}\left(\mathrm{S}, \mathrm{f}_{1}, \mathrm{f}_{2}\right)$ obtained with this procedure were unbiased even down to 16 trials per stimulus, a value smaller than the number of trials per stimulus obtained in the experiments considered here (Suppl. Fig 1). Therefore, this conservative binning and bias subtraction procedure gave robustly unbiased estimates of estimates $\mathrm{I}(\mathrm{S}, \mathrm{f})$ and $\mathrm{I}\left(\mathrm{S}, \mathrm{f}_{1}, \mathrm{f}_{2}\right)$ that did not artificially affect redundancy calculations due to sampling bias effects, although binning into only $n=4$ bins underestimated the total information in the LFP responses, by approximately $20 \%$ (see Supplemental Material, section "Simulated LFP data"). We however verified (data not shown) that increasing the number of bins up to six (the maximum that lead to relatively unbiased calculations of I $\left(\mathrm{S}, \mathrm{f}_{1}, \mathrm{f}_{2}\right)$ with the current number of trials, see(Magri et al. 
2009)) gave almost identical results and did not change any of the conclusions about which bands are informative and whether they are redundant. We refer to (Belitski et al. 2008; Kayser et al. 2009; Montemurro et al. 2008) for further details and tests of the analysis procedures.

\subsection{Coefficient of variation}

To quantify the trial to trial variability of the LFP energy we computed the coefficient of variation (CV). This is defined as the ratio of the variance to the mean, both computed across trials and provides a normalized measure of variability. Large values indicate more variable signals.

\subsection{Signal and noise correlations across frequency bands}

The information analysis tells which frequency ranges allow better discrimination among stimuli on a single trial, but it does not tell whether the information increase at certain frequencies is due to greater reliability across trials of the responses at these frequencies, or due to improved stimulus differentiation by the mean responses. To separate out the contribution of stimulus modulation and of response variability, it is useful to characterize the response $r_{f}$ in each stimulus window as 'signal plus noise' (Averbeck et al. 2006; Gawne and Richmond 1993; Panzeri et al. 1999):

$r_{f}=\bar{r}_{f}+n_{f}$

where the "signal" $\bar{r}_{f}$ is defined by the trial-averaged power (the bar denotes the average across trials at fixed stimulus), and the "noise" is defined by the trial-by-trial fluctuations $n_{f}$ of the response around their mean value across trials. Importantly, "noise" here reflects different possible response variations at fixed stimulus, whatever their origin (for example, variations induced by the effect of ongoing activity, by stimulus-independent neuromodulation or by the activity of other neurons projecting to this area and not driven by the considered stimuli, and others).

To determine which frequencies have related stimulus selectivity and which have shared sources of variability, we analyzed signal and noise correlations was follows: The "signal" correlation between two frequency bands is defined as the correlation of the mean (i.e. trial-averaged) responses across different stimuli. Positive values indicate that the two frequencies have similar stimulus preference, whereas small values indicate that two frequencies prefer uncorrelated stimuli. Correlations that manifest as covariations of the trial-by-trial fluctuation around the mean response are traditionally called "noise correlations". Since these noise covariations are measured at fixed stimulus, they ignore all effects attributable to shared stimulation or shared stimulus preferences. To quantify the strength of noise correlations, we computed the Pearson correlation coefficient (across trials at fixed stimulus) of the trialaverage-subtracted powers $n_{f 1}$ and $n_{f 2}$, and then we averaged this over all stimulus windows. This quantifies the correlations of the variations around the mean at each trial and stimulus window. Positive values of noise correlation indicate that when the power of one frequency fluctuates over its mean values, the power in the other frequency is also more likely to do so.

\section{Results}

\subsection{LFP responses to dynamic naturalistic stimuli}

The amplitude of field potentials in sensory cortices is modulated by time varying naturalistic stimuli. This has been recognized in previous studies (Belitski et al. 2008; Kayser et al. 2009; Kayser et al. 2003b; Montemurro et al. 2008) and is exemplified in Fig. 1(b) for one electrode in auditory cortex. The example trace illustrates the raw LFP together with the band-pass filtered signal $(4-8 \mathrm{~Hz})$ and the envelope for a single repeat of the stimulus. That the sensory stimulus reliably modulates the LFP can be seen in the lowest panel, were the same energy value consistently lines up over trials (rows) at several instances (see arrows). Such reliable LFP responses were frequent in the entire dataset and demonstrate the 'driving' influence of sensory stimuli on LFPs in sensory cortices.

\subsection{Stimulus information in LFPs}

We used information theory to systematically quantify which LFP bands are reliably related to naturalistic stimuli. Following previous studies (de Ruyter van Steveninck et al. 1997; Strong et al. 1998) we used a general stimulus definition, which captures the information carried about all possible features appearing in the stimulus preceding the response, without making specific assumptions about the nature of these features and the specific 'latency' between stimulus and response (see Materials and Methods for details). The stimulus presentation time was divided into nonoverlapping windows of length $T$, each window was assigned a different stimulus number $s_{i}$ (Fig. 1a). This formulation has the advantage that it can be applied in the very same form to different neural signals and sensory stimuli, and hence provides a principled assessment of the stimulus relatedness of LFPs in different systems. The stimulus window length $T$ is a free parameter of the analysis. Choosing its value and computing the corresponding information corresponds to formulating and evaluating a hypothesis about the time precision with which the neural signals can tell apart different sections of the dynamic stimulus. In visual cortex, 
we previously concentrated only on encoding of visual features by LFP energy at slow time scales ( $2 \mathrm{~s}$, (Belitski et al. 2008)), whereas in auditory cortex we focused only on encoding of natural sounds by LFP energy on very fast time scales (few milliseconds (Kayser et al. 2009)). In the following, we extend these studies by performing a parametric analysis of how information carried by different LFP frequencies depends on the time scale $T$. Such analysis not only provides insights into the relevant time scales of the neural signal, but also about the time scales at which the sensory features reflected in the LFPs might vary.

Figure 2(a) displays the stimulus information in the LFP energy as a function of frequency band $f$ and stimulus window $T$. Several common properties of both datasets become apparent. First, the lowest frequencies provide the highest stimulus information for many of the tested window lengths, demonstrating that slow field potentials are strongly stimulus driven. Second, the overall information increases with increasing window length, showing that the time-averaged LFP energy is more informative than the same signal considered on a very short time scale. And third, additional peaks of high information become evident in higher frequency bands at the longest windows investigated: in both datasets information in the gamma band $(>50 \mathrm{~Hz})$ is most apparent in the longest $(2048 \mathrm{~ms})$ window, demonstrating that especially the high frequency bands gain information when considered on longer time scales (c.f. also Fig. 2b). However, stimulus information in gamma frequency bands $(>50 \mathrm{~Hz})$ was considerably higher in the visual compared to the auditory dataset, when compared to stimulus information in low frequency bands. For the longest time window considered $(T=2048 \mathrm{~ms})$, information in gamma bands reached only about $40 \%$ of the information in the lowest frequency bands for the auditory dataset, but nearly $110 \%$ for the visual data.

To provide a more intuitive understanding of the time scales on which the LFP energy was computed for the above analysis, we indicated the number of cycles of each LFP band that fit within one time window in the lower panel of Fig. 2(a). For low frequency bands and short time windows only a fraction of a cycle was considered for estimating the energy, while for long windows and high frequency bands a large number of cycles was averaged. It should be noted that by virtue of filtering and envelope extraction, the energy of a particular frequency band $f$ can only vary on time scales slower than this frequency (i.e. time windows longer than one oscillation cycle). That the stimulus information does not depend much on the time window is hence not surprising for the lowest frequency bands (e.g. $0-4 \mathrm{~Hz}$ ). For higher frequency bands however, energy and stimulus information could readily depend on the length of the time window. For the $50-54 \mathrm{~Hz}$ band, as an example, energy (and stimulus information) could vary on time scales longer than $20 \mathrm{~ms}$. That stimulus information increases with increasing time windows for most of the frequency bands is hence a genuine result and demonstrates that the LFP is most informative when the energy of several oscillation cycles is averaged.

\subsection{Trial to trial reliability and time scales}

To elucidate why stimulus information increases with increasing window length we quantified the trial to trial reliability of the LFP energy by computing its coefficient of variation. The coefficient of variation is defined as the ratio of the standard deviation of the LFP energy across trials divided by the mean and provides a scale-free measure of variability. In general, high information values result from good separability of the different stimuli, which profits from both, distinct mean responses and high trial by trial reliability. Of these, the coefficient of variation assesses the latter. The result in Fig. 2(c) reveals a drop in variability with increasing time window, in good agreement with those bands gaining most information. This suggests that the increasing information in the LFP on long time scales results from increased reliability of the LFP energy when pooled over several cycles of the respective oscillation. In addition, the results also reveal high variability at low frequency bands. That these bands carry large amounts of information hence suggests that different time epochs elicit slow LFPs with largely varying amplitudes.

The increase in reliability of different frequency bands with increasing time scale is exemplified in Fig. 2(d,e). The low frequency example (4-8 $\mathrm{Hz}$ band) reveals high trial to trial consistency (Fig. 2d, panel b) and when quantified on short (panel c, $T=4 \mathrm{~ms}$ ) and long (panel d, T=1024 ms) time scales the envelope of several trials shows good overlap. The high frequency example (56-60 Hz band), in contrast, shows little consistency between the two example trials (panels a,b). When quantified in short windows $(\mathrm{T}=$ $4 \mathrm{~ms}$ ) this band reveals little overlap of the envelope extracted from different trials (panel c). However, when quantified in longer windows ( $\mathrm{T}=1024 \mathrm{~ms})$ many instances of high trial to trial reliability become apparent (panel d). Supplemental Figure 2 illustrates the same point using histograms. This lets us conclude that the increasing stimulus information in the LFP energy reflects increased reliability of the LFP energy when considered on longer time scales.

\subsection{LFP modulation and stimulus information}

The above reveals that the spectral structure of stimulus information differs between the visual and auditory datasets, especially in the high frequency $(>50 \mathrm{~Hz})$ regime. On investigating the source of this, we asked whether there is a 

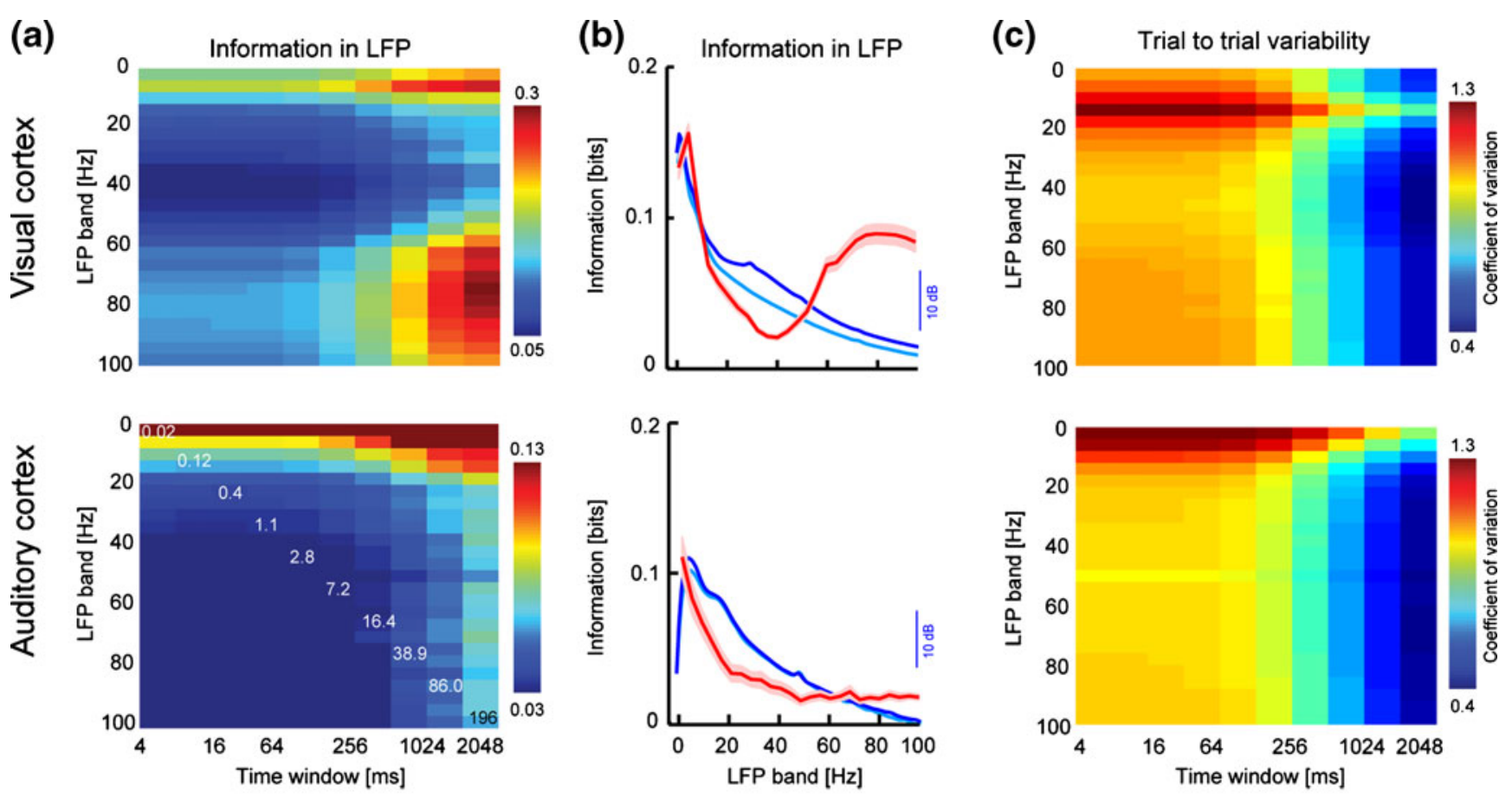

(d)
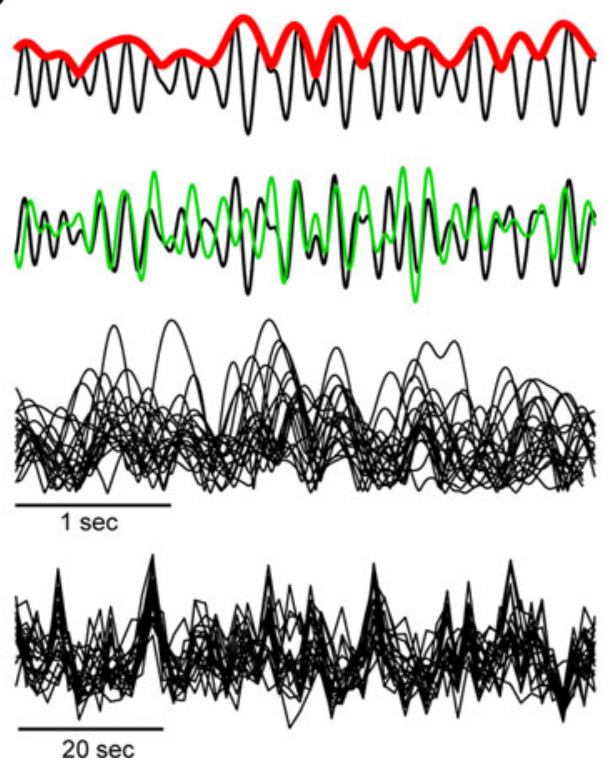

Fig. 2 Stimulus information on different time scales. (a) Stimulus information (units of bits) in the LFP energy of different frequency bands and for time windows $T$ of different length. Each row displays the mean value for the respective dataset. White numbers in the lower panel indicate the number of cycles of the respective band included in one time window. Note that the scales for auditory and visual data differ. (b) Information for intermediate time windows $(\mathrm{T}=128 \mathrm{msec})$. Red lines denote the mean, shading the s.e.m across recording sites. Blue lines show the power of the LFP signal as a function of frequency, averaged for the stimulation period (dark) and a pre- (e)

(a)

(b)

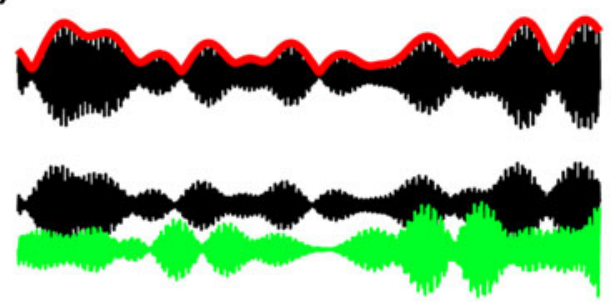

(c)

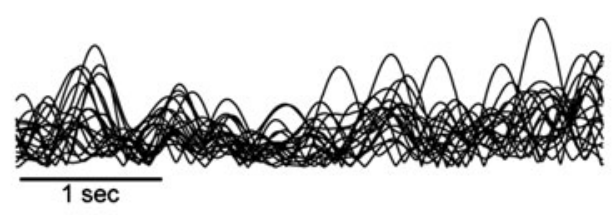

(d)

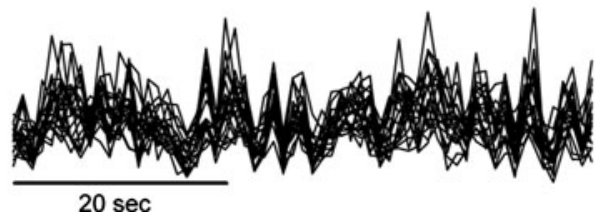

stimulus baseline period (light). (c) Coefficient of variation of the LFP energy across trials. The coefficient is defined as the ratio of the standard deviation (across trials) to the mean, and lower values indicate a lower variability. Graphs display the mean for each dataset. (d) Example data from visual cortex the 4-8 Hz band. a) Single trial band-pass filtered signal together with envelope. b) Two single trials. c) Envelope of multiple trials computed in $4 \mathrm{~ms}$ time windows. d) Envelope of multiple trials computed in $1024 \mathrm{~ms}$ windows. (e) Same as in $\mathrm{D}$, but for the $56-60 \mathrm{~Hz}$ band 
relation between the spectral structure of stimulus information and the spectral structure of the stimulus induced LFP energy modulation. Previous studies have shown that the LFP energy is modulated by sensory stimuli to varying degrees in different bands, and have used this energy modulation as a index characterizing the tuning of LFPs to different sensory features (Kayser et al. 2007; Kayser et al. 2003b; Siegel and Konig 2003). Figure 2(b) displays the average power of the LFP signal during sensory stimulation (dark blue) and during pre-stimulus baseline periods (light blue, absence of the specific sensory stimulus). In the auditory data, the LFP power modulation (difference between power during stimulus and baseline periods) was highest at the lowest frequencies and decreased in higher bands. In the visual data, the power modulation was strongest at frequencies around $30 \mathrm{~Hz}$ (the frame rate of the visual stimulus), but in general the power was increased for all frequencies above about $20 \mathrm{~Hz}$.

To quantify the relation between the spectral structure of stimulus information and the modulation of the LFP power by the sensory stimulus, we used correlation analysis. In detail, for each electrode we computed the Pearson correlation of the energy modulation and the stimulus information across frequencies ( $n=25$ bins), and averaged the correlations across electrodes. The resulting values were small for both datasets (visual cortex: $r=-0.2 \pm 0.08$; auditory cortex: $r=0.1 \pm 0.02$, mean and s.e.m.), demonstrating that the frequency dependence of the stimulus information cannot be explained by the frequency dependence of the energy modulation. As a result, the difference between the spectral distribution of stimulus information in the visual and auditory datasets is not the immediate result of distinct power modulation induced by the stimulus, but must rather reflect distinct mechanisms of how stimulus drive is converted into reliably stimulus associated field potential modulations.

\subsection{Synergy between LFP bands}

An important question with regard to exploiting LFPs in applications such as brain decoding is whether different LFP bands, or LFPs and multi-unit activity (MUA), provide similar or redundant information about sensory stimuli (Donoghue 2008; Nicolelis and Lebedev 2009). To quantify the similarity of different bands in encoding stimulus information, we computed the synergy between pairs of frequency bands. For the visual data, such an analysis has already been presented in a previous study (Belitski et al. 2008), but not so for the auditory data.

Figure 3(a) displays the joint information in pairs of frequency bands for both datasets (using $T=2048 \mathrm{~ms}$ windows), which mostly replicated the pattern found for individual bands before: High information in low frequency bands for both datasets and an additional informative peak above $50 \mathrm{~Hz}$ in the visual data. The overall synergy between bands was small (Fig. 3b), suggesting that most frequencies carried independent information about the stimulus. The strongest deviation from independence was found for directly neighboring, and for high frequency bands. For bands above $50 \mathrm{~Hz}$, we found negative synergy (i.e. redundancy) between frequencies, which was on the order of up to $20 \%$ of the total information. Noteworthy, when computed in shorter time windows, redundancy was even smaller. This information independence between frequency bands is compatible with previous results (Belitski et al. 2008) and implies that a separation of the LFP into smaller frequency bands is indeed useful, because each band adds almost independent information about the sensory stimulus.

Inspecting example data again provides an intuitive basis of this result. Figure 3(c) displays the (trial averaged) LFP energy for different frequency bands for one recording site in auditory cortex. While both high frequency bands (red, orange traces) show very similar dynamics, the low frequency band (black trace) deviates from this pattern and exhibits a more distinct time course. Overall, this suggests that high frequency bands are redundant because they share a very similar temporal dynamics (stimulus drive), which differs from that outlined by much slower bands.

Further insight into why different frequency bands are largely independent is provided by analysis of signal and noise correlations. Signal correlations characterize the similarity in stimulus preference between two signals, while noise correlations characterize shared covariations on a trial by trial basis around the mean response. The results displayed in Fig. 4 reveal that while neighboring frequency bands share similarities in stimulus preference (signal correlations), separated bands are independent in their preferences. A similar result holds for noise correlations: shared trial by trial variability is strongest for neighboring frequency bands, although the noise correlations were much weaker than signal correlations. These findings suggest that the information independence between low and high frequency bands is a result of the different stimulus preferences; the rather small redundancy between different high frequency bands results from small differences in tuning and small noise correlations.

\subsection{Synergy between LFP bands and spikes}

Combining different LFP bands with MUA recorded on the same electrode also provided largely independent information (Fig. 5). Synergy was minimal especially for short time windows and in low frequency bands, while for longer time windows and high frequency bands $(>50 \mathrm{~Hz})$ there was 
Fig. 3 Information synergy between LFP bands. (a) Joint information provided by the energy of pairs of LFP bands (mean values across sites). (b) Synergy between pairs of bands, expressed as percent of the sum of the information of both bands considered independently. Negative values (blue) indicate redundancy. (c) Example data from auditory cortex, displaying the mean LFP energy from one recording site and three different bands (sampled at $\mathrm{T}=2048 \mathrm{~ms}$ )

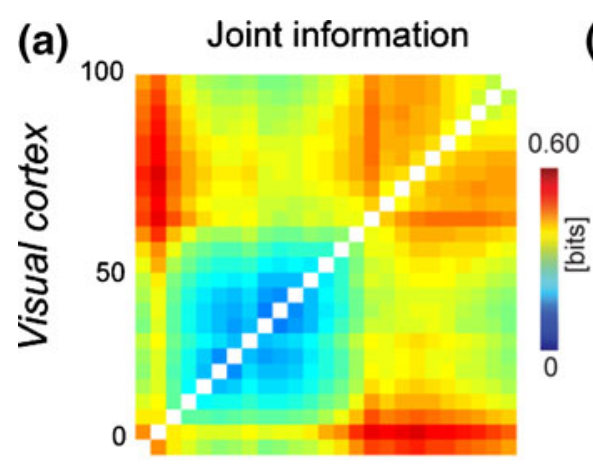

(b) Synergy
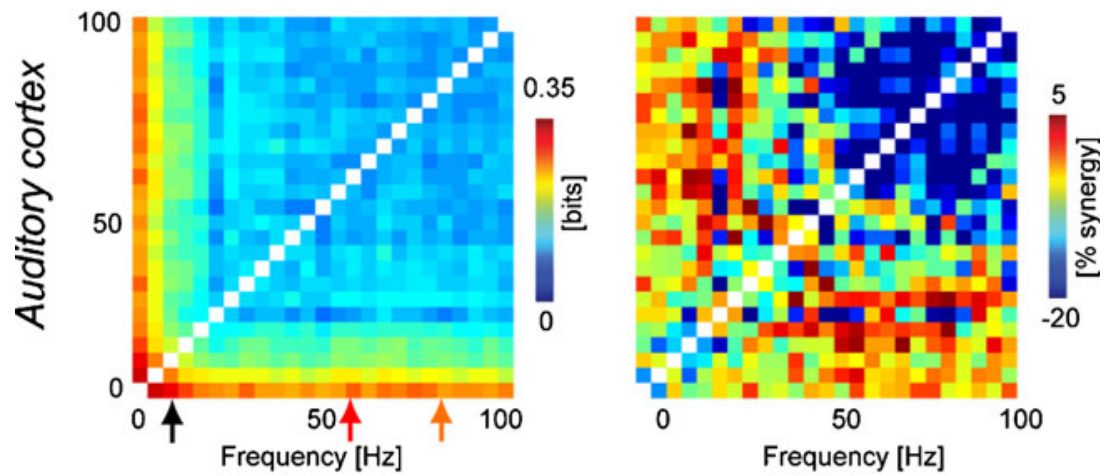

(c)

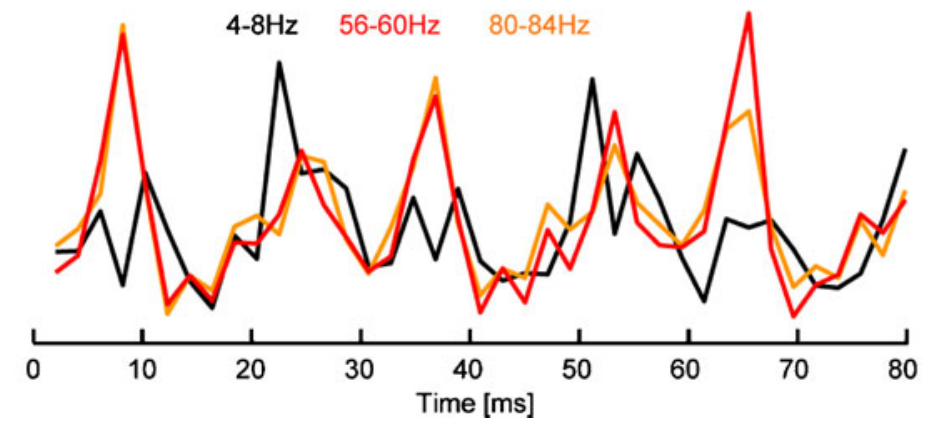

some redundancy. Still, this redundancy was small and accounted for less than $10 \%$ of the information. This differing dependency between low and high frequency LFP bands and spiking activity is also exemplified in Fig. 5(c) by example data from visual cortex. The time courses of the 4-8 Hz band and MUA differ more than those of the 68$72 \mathrm{~Hz}$ band and MUA. Overall this demonstrates that spiking activity is more related to high frequency bands $(>50 \mathrm{~Hz})$, but overall provides largely complementary stimulus information than the LFP energy.

\section{Discussion}

The cortical LFP is a mesoscopic signal of neural activity, which reflects aspects of sensory processing and higher cognitive functions. Despite great interest in this signal in the context of 'brain decoding', only few studies have systematically investigated stimulus information in sensory cortical LFPs. As a result, the origin of the LFP, its relation to neuronal spiking activity, and the degree to which different frequency bands are stimulus related are still a matter of debate. To shed light on these questions, we extended approaches developed in previous studies (Belitski et al. 2008; Kayser et al. 2009) and performed a systematic analysis of stimulus information in LFPs recorded from visual and auditory cortices. For our analysis we relied on information theoretic methods, which provide a principled approach to quantify the relation between indices of neural activity and external stimuli (Borst and Theunissen 1999; Jacobs et al. 2009; Quian Quiroga and Panzeri 2009).

\subsection{Spectral structure of informative frequency bands}

Analyzing the spectral distribution of stimulus information revealed that low frequency bands $(10 \mathrm{~Hz})$ were strongly stimulus related, while intermediate frequency bands (10 to 

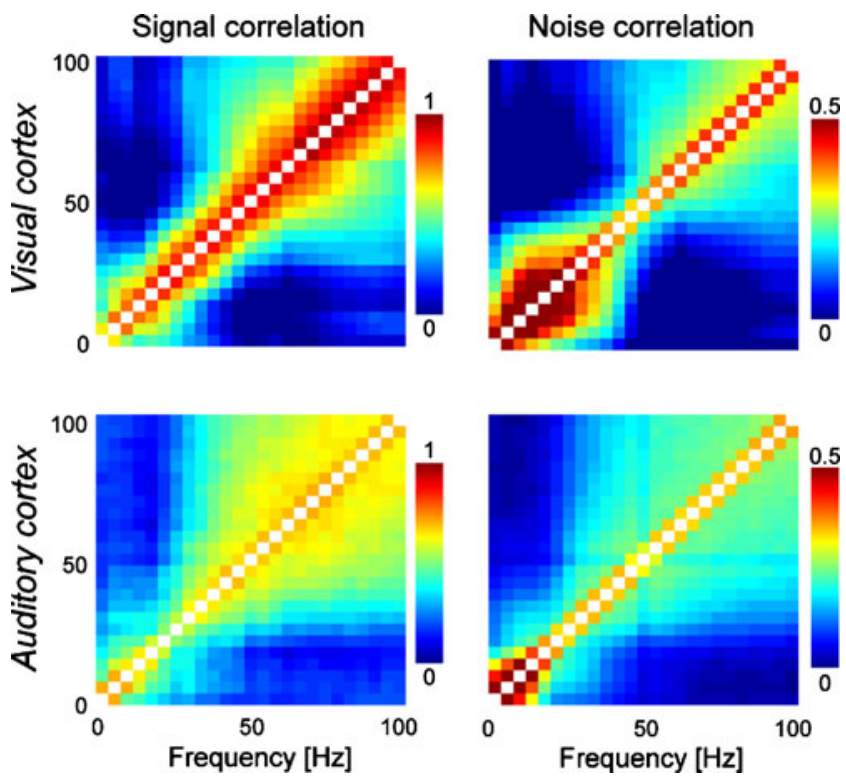

Fig. 4 Signal and noise correlations. This figure displays the signal and noise correlations between pairs of frequency bands for both datasets (2048 ms time windows). Each panel shows the average across sites. Please refer to the Main Text or the Methods for a definition of signal and noise correlations
$50 \mathrm{~Hz}$ ) were so to a much smaller degree. This fits well with the notion that slow oscillations in sensory cortices are reliably entrained by dynamic stimuli, a mechanism that is likely to play an important role for the processing of dynamic naturalistic stimuli (Kayser et al. 2009; Lakatos et al. 2008; Luo and Poeppel 2007; Schroeder and Lakatos 2009). Stimulus information in higher (gamma, $>50 \mathrm{~Hz}$ ) frequency bands revealed a second peak, which was considerably more prominent in the visual dataset. Whether this difference between datasets arises from the different preparations (alert vs. anaesthetized animals) or reflects a more basic difference between sensory systems cannot be determined with the present dataset and remains to be elucidated in future studies. Nonetheless, this stimulus related gamma band conforms to the notion that gamma oscillations participate in sensory coding, especially in the visual system (Berens et al. 2008; Fries et al. 2007; Kayser and Konig 2004; Siegel and Konig 2003).

Stimulus information depends more on the reliability of a signal associated with a particular stimulus, and much less on the overall signal amplitude. In concordance with this, our results demonstrate that the amount of information
Fig. 5 Information synergy between LFP bands and MUA. (a) Synergy between LFP and MUA as a function of frequency band and time scale. Synergy is expressed as percent of the sum of the information of both bands considered independently. Negative values (blue) indicate redundancy. (b) Synergy between LFP bands and MUA when both are computed in $2048 \mathrm{~ms}$ windows. (c) Example data from visual cortex showing the similar (trial averaged) time courses of MUA and high frequency LFPs $(60-64 \mathrm{~Hz})$ and the different time course of a low frequency band (4-8 Hz, sampled at $\mathrm{T}=1024 \mathrm{~ms}$ )

\section{(a) \\ 要}
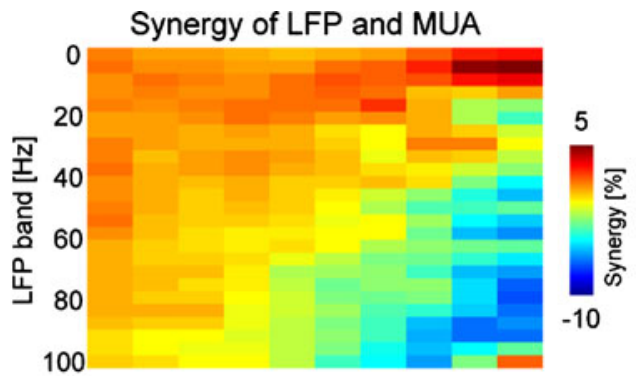

(b) Synergy of LFP and MUA

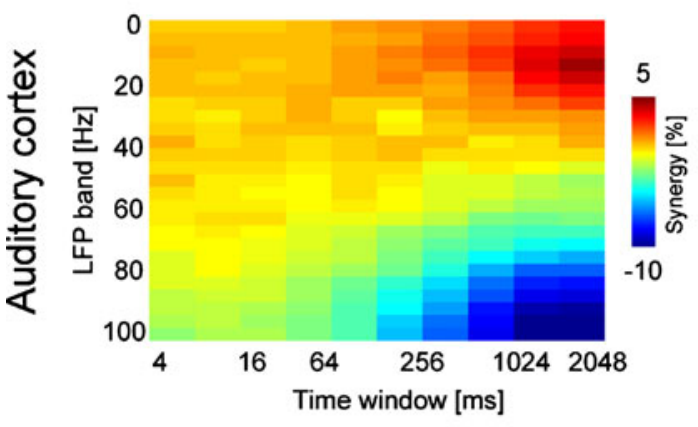

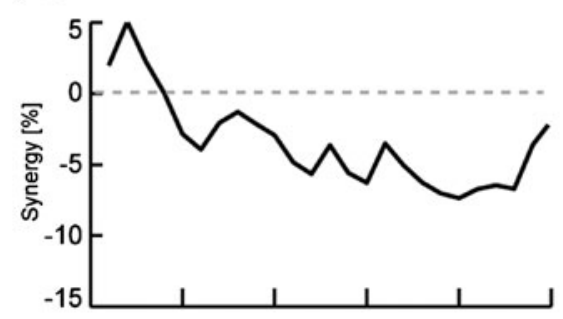

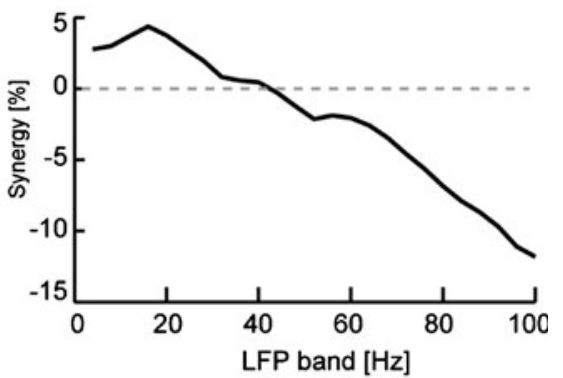

(c)
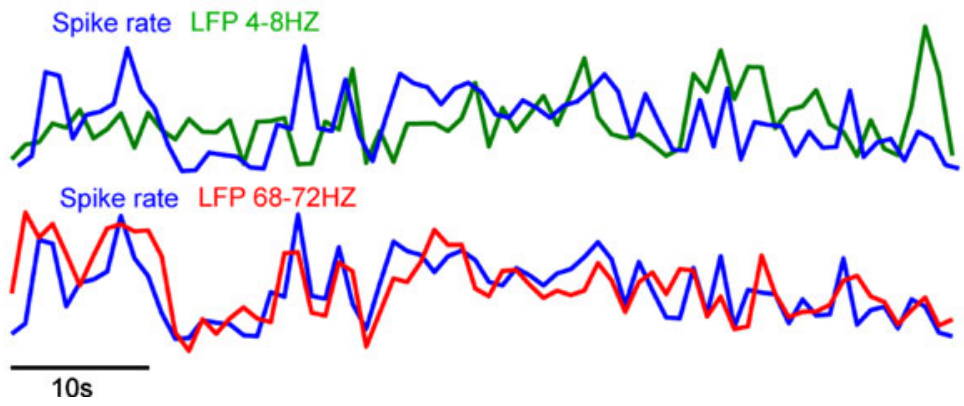
carried by a particular frequency band cannot be predicted from the LFP power in the considered band. Analyzing LFPs just in terms of signal strength can hence be misleading when used to draw conclusions about the ability of different bands to represent sensory stimuli. More principled methods such as stimulus decoding or information theory provide a more suitable approach to elucidate this.

\subsection{Time scales of stimulus information}

A major focus of our analysis was to elucidate the time scales on which different LFP bands are informative. We found that for all frequency bands stimulus information increased with increasing length of the time window $\mathrm{T}$. The increase was small for slow oscillations that vary on a time scale similar to the time windows considered (e.g. the 1$4 \mathrm{~Hz}$ band), and was much larger for higher frequency bands $(>50 \mathrm{~Hz})$. This finding demonstrates that the slow envelope of fast oscillations, i.e. the average amplitude of many (hundreds of) cycles of the respective oscillation, is much more stimulus related than the instantaneous amplitude of individual cycles. Hence, for higher frequency bands the individual cycle of the oscillation plays a much smaller role in representing the sensory stimulus than for lower frequency bands, for which even the amplitude of a single cycle (or less) is stimulus informative.

Our results demonstrate that the increased information on longer time scales results from increased trial to trial reliability of the LFP energy in longer time windows. This suggests that the energy (amplitude) of individual oscillation cycles is much less reliably affected by the stimulus than the signal obtained by pooling the energy of multiple oscillation cycles. That LFPs become more informative on longer time scales might hence be a consequence of the biophysical processes generating the amplitude of individual LFP cycles. Yet, it should be noted that the finding of individual gamma cycles carrying little stimulus information does not preclude individual gamma cycles from playing a role in 'grouping' the responses of different neurons or in routing information from one area to another (Fries et al. 2007; Womelsdorf and Fries 2007; Womelsdorf et al. 2006). In fact, to be able to dynamically group or route information, it seems rather advantageous if the timing or amplitude of individual cycles is more controlled by intrinsic mechanisms of the brain than by the external stimulus.

In addition, our result also suggests that the LFP likely reflects slow features in the sensory stimulus. If the LFP energy in a particular band was to reflect fast stimulus properties that change on the scale of a few (tens of) milliseconds, then averaging the neural signal over considerably longer time scales would likely diminish the representation of this particular stimulus property. That stimulus information increases on longer time scales rather suggests that the LFP energy reflects more slowly changing stimulus attributes, which are very prominent in natural stimuli (Dong and Atick 1995; Kayser et al. 2003a; Singh and Theunissen 2003).

\subsection{Relation between LFP bands and spiking activity}

Our analysis revealed that individual (4 $\mathrm{Hz}$ spaced) frequency bands carry largely independent information about natural stimuli and also carry information largely independent to that provided by neuronal spiking activity (MUA). This information independence of different bands justifies the partitioning of the frequency axis into distinct and functionally independent bands (Buzsaki 2006; Roopun et al. 2008; Steriade 2006). Often a division of the frequency axis follows the prominent frequencies found in the human EEG, or a specific division related to the particular features or variables of interest. Our results support the notation that this division is not only empirical, but reflects a true functional decoupling of different frequency regimes which is conserved across sensory systems. While such an independence of different LFP bands has been reported previously for visual cortical LFPs (Belitski et al. 2008), the present study demonstrates that this result also holds on different time scales and in another sensory modality.

Our study of linear correlation of stimulus preference ("signal correlation") between the energy of different bands is in agreement with previous studies based on linear correlation analysis reporting that the energy in neighboring LFP bands tends to respond to similar (but not equal) values of a stimulus feature (Berens et al. 2008; Kayser and Konig 2004; Siegel and Konig 2003). At first, this might seem at odds with the small or negligible amount of redundancy that we found using an information theoretic measure rather than a liner correlation of trial-averaged responses. To reconcile these different views, one should note that information based analysis not only considers preferred stimuli, but also other aspects such as response reliability or signal and noise correlations (Averbeck et al. 2006; Panzeri et al. 2003), which are not taken into account by linear correlations. In fact, our correlation analysis directly demonstrates that nearby frequency bands share similar stimulus preferences and exhibit strong signal correlations. However, noise correlations, which can either enhance or reduce the redundancy between frequency bands, are weak, implying that while different bands prefer related stimuli on average, they exhibit decoupled trial by trial variability. Considering the present findings, we hence conclude that separate LFP bands provide largely independent information channels, which when combined, can 
greatly enhance the capacities in practical applications such as brain machine interfaces (Donoghue 2008).

4.4 Potential mechanisms generating the spectral dependence of LFP information

Our results demonstrate that the low frequency LFPs are especially reliable in response to naturalistic stimuli and their energy is well informative about them. Understanding the mechanisms generating such stimulus representations in low frequency LFPs is hence of great interest, and a recent modeling study can provide important insights. Studying the dynamics of recurrent network models of inhibitory and excitatory neurons, Mazzoni and colleagues (Mazzoni et al. 2008) found that slow components of network LFPs arise from stimulus to neural interactions, i.e. from the direct drive of slow fluctuations in the input to the principal neurons. Since changes in sensory features within naturalistic stimuli (such as the ones used in our experiments) are known to vary slowly and are characterized by temporal regularities with maximal power in the lowest frequencies (Dong and Atick 1995; Kayser et al. 2003a; Singh and Theunissen 2003), this hypothesis would predict that LFPs would follow stimulus dynamics principally at the corresponding slow frequencies. In other words, prominent slow changes in the stimulus may elicit corresponding slow shifts in network excitability. This hypothesis could not only explain why low frequency LFPs are informative about naturalistic stimuli, but is also consistent with other findings reporting the entrainment of low-frequency cortical LFPs to stimuli with strong temporal regularities (Schroeder and Lakatos 2009; Schroeder et al. 2008). Studying the dynamics of recurrent network models of inhibitory and excitatory neurons also revealed that faster components of network LFPs are determined by neural to neural interactions, i.e. by the coupling of different populations of (excitatory and inhibitory) neurons (Brunel and Wang 2003; Mazzoni et al. 2008). Importantly, in this modeling study the strength of these faster LFPs was not modulated by the stimulus dynamics, but by the overall strength of the stimulus, hence a static property. These theoretical results hence suggest that stationary properties are more likely to impact on fast oscillations, while slow naturalistic dynamic stimulus properties are more likely reflected in low frequency LFPs. This conclusion seems well compatible with our experimental findings.

Acknowledgements This work was supported by the Max Planck Society and by the BMI project of the Italian Institute of Technology. We would like to thank the participants of the "Modeling Extracellular Potentials" Workshop for inspiring discussions.
Open Access This article is distributed under the terms of the Creative Commons Attribution Noncommercial License which permits any noncommercial use, distribution, and reproduction in any medium, provided the original author(s) and source are credited.

\section{References}

Averbeck, B. B., Latham, P. E., \& Pouget, A. (2006). Neural correlations, population coding and computation. Nature Reviews. Neuroscience, 7(5), 358-366.

Bartos, M., Vida, I., \& Jonas, P. (2007). Synaptic mechanisms of synchronized gamma oscillations in inhibitory interneuron networks. Nature Reviews. Neuroscience, 8(1), 45-56.

Belitski, A., Gretton, A., Magri, C., Murayama, Y., Montemurro, M. A., Logothetis, N. K., et al. (2008). Low-frequency local field potentials and spikes in primary visual cortex convey independent visual information. Journal of Neuroscience, 28 (22), 5696-5709.

Berens, P., Keliris, G. A., Ecker, A. S., Logothetis, N. K., \& Tolias, A. S. (2008). Comparing the feature selectivity of the gammaband of the local field potential and the underlying spiking activity in primate visual cortex. Frontiers in Systems Neuroscience, 2, 2.

Borst, A., \& Theunissen, F. E. (1999). Information theory and neural coding. Nature Neuroscience, 2(11), 947-957.

Brunel, N., \& Wang, X. J. (2003). What determines the frequency of fast network oscillations with irregular neural discharges? I. Synaptic dynamics and excitation-inhibition balance. Journal of Neurophysiology, 90(1), 415-430.

Buzsaki, G. (2002). Theta oscillations in the hippocampus. Neuron, 33 (3), 325-340.

Buzsaki, G. (2006). Rhythms of the brain. Oxford University Press.

Buzsaki, G., Bickford, R. G., Ponomareff, G., Thal, L. J., Mandel, R., \& Gage, F. H. (1988). Nucleus basalis and thalamic control of neocortical activity in the freely moving rat. Journal of Neuroscience, 8(11), 4007-4026.

Cardin, J. A., Carlen, M., Meletis, K., Knoblich, U., Zhang, F., Deisseroth, K., et al. (2009). Driving fast-spiking cells induces gamma rhythm and controls sensory responses. Nature.

de Ruyter van Steveninck, R. R., Lewen, G. D., Strong, S. P., Koberle, R., \& Bialek, W. (1997). Reproducibility and variability in neural spike trains. Science, 275(5307), 1805-1808.

Dong, D. W., \& Atick, J. J. (1995). Statistics of natural time varying images. Network: Computation in Neural Systems, 6(3), 345-358.

Donoghue, J. P. (2008). Bridging the brain to the world: a perspective on neural interface systems. Neuron, 60(3), 511-521.

Frien, A., Eckhorn, R., Bauer, R., Woelbern, T., \& Gabriel, A. (2000). Fast oscillations display sharper orientation tuning than slower components of the same recordings in striate cortex of the awake monkey. European Journal of Neuroscience, 12(4), 1453-1465.

Fries, P., Nikolic, D., \& Singer, W. (2007). The gamma cycle. Trends in Neurosciences, 30(7), 309-316.

Gawne, T. J., \& Richmond, B. J. (1993). How independent are the messages carried by adjacent inferior temporal cortical neurons? Journal of Neuroscience, 13(7), 2758-2771.

Hatsopoulos, N. G., Ojakangas, C. L., Paninski, L., \& Donoghue, J. P. (1998). Information about movement direction obtained from synchronous activity of motor cortical neurons. Proceedings of the National Academy of Sciences of the United States of America, 95(26), 15706-15711.

Jacobs, A. L., Fridman, G., Douglas, R. M., Alam, N. M., Latham, P. E., Prusky, G. T., et al. (2009). Ruling out and ruling in neural codes. 
Proceedings of the National Academy of Sciences of the United States of America, 106(14), 5936-5941.

Juergens, E., Guettler, A., \& Eckhorn, R. (1999). Visual stimulation elicits locked and induced gamma oscillations in monkey intracortical- and EEG-potentials, but not in human EEG. Experimental Brain Research, 129(2), 247-259.

Kayser, C., \& Konig, P. (2004). Stimulus locking and feature selectivity prevail in complementary frequency ranges of $\mathrm{V} 1$ local field potentials. European Journal of Neuroscience, 19(2), 485-489.

Kayser, C., Einhäuser, W., \& König, P. (2003). Temporal correlations of orientations in natural scenes. Neurocomputing, 52, 117-123.

Kayser, C., Salazar, R. F., \& Konig, P. (2003). Responses to natural scenes in cat V1. Journal of Neurophysiology, 90(3), 1910-1920.

Kayser, C., Petkov, C. I., \& Logothetis, N. K. (2007). Tuning to sound frequency in auditory field potentials. Journal of Neurophysiology, 98(3), 1806-1809.

Kayser, C., Montemurro, M. A., Logothetis, N., \& Panzeri, S. (2009). Spike-phase coding boosts and stabilizes the information carried by spatial and temporal spike patterns. Neuron, 61, 597-608.

Lakatos, P., Karmos, G., Mehta, A. D., Ulbert, I., \& Schroeder, C. E. (2008). Entrainment of neuronal oscillations as a mechanism of attentional selection. Science, 320(5872), 110-113.

Liu, J., \& Newsome, W. T. (2006). Local field potential in cortical area MT: stimulus tuning and behavioral correlations. Journal of Neuroscience, 26(30), 7779-7790.

Logothetis, N. K. (2002). The neural basis of the blood-oxygen-leveldependent functional magnetic resonance imaging signal. Philosophical Transactions of the Royal Society of London. Series B: Biological Sciences, 357(1424), 1003-1037.

Logothetis, N. K. (2008). What we can do and what we cannot do with fMRI. Nature, 453(7197), 869-878.

Luo, H., \& Poeppel, D. (2007). Phase patterns of neuronal responses reliably discriminate speech in human auditory cortex. Neuron, 54(6), 1001-1010.

Magri, C., Whittingstall, K., Singh, V., Logothetis, N. K., \& Panzeri, S. (2009). A toolbox for the fast information analysis of multiplesite LFP, EEG and spike train recordings. BMC Neuroscience, 10,81

Mazzoni, A., Panzeri, S., Logothetis, N. K., \& Brunel, N. (2008). Encoding of naturalistic stimuli by local field potential spectra in networks of excitatory and inhibitory neurons. PLoS Computational Biology, 4(12), e1000239.

Mitzdorf, U. (1985). Current source-density method and application in cat cerebral cortex: investigation of evoked potentials and EEG phenomena. Physiological Reviews, 65(1), 37-100.

Montemurro, M. A., Senatore, R., \& Panzeri, S. (2007). Tight datarobust bounds to mutual information combining shuffling and model selection techniques. Neural Computation, 19(11), 29132957.

Montemurro, M. A., Rasch, M. J., Murayama, Y., Logothetis, N. K., \& Panzeri, S. (2008). Phase-of-firing coding of natural visual stimuli in primary visual cortex. Current Biology, 18(5), 375380

Nicolelis, M. A., \& Lebedev, M. A. (2009). Principles of neural ensemble physiology underlying the operation of brain-machine interfaces. Nature Reviews. Neuroscience, 10(7), 530-540.
Panzeri, S., Schultz, S. R., Treves, A., \& Rolls, E. T. (1999). Correlations and the encoding of information in the nervous system. Proceedings in Biological Sciences, 266(1423), 1001-1012.

Panzeri, S., Pola, G., \& Petersen, R. S. (2003). Coding of sensory signals by neuronal populations: the role of correlated activity. Neuroscientist, 9(3), 175-180.

Panzeri, S., Senatore, R., Montemurro, M. A., \& Petersen, R. S. (2007). Correcting for the sampling bias problem in spike train information measures. Journal of Neurophysiology, 98(3), 10641072 .

Pettersen, K. H., \& Einevoll, G. T. (2008). Amplitude variability and extracellular low-pass filtering of neuronal spikes. Biophysical Journal, 94(3), 784-802.

Quian Quiroga, R., \& Panzeri, S. (2009). Extracting information from neuronal populations: information theory and decoding approaches. Nature Reviews. Neuroscience, 10(3), 173-185.

Rasch, M. J., Gretton, A., Murayama, Y., Maass, W., \& Logothetis, N. K. (2008). Inferring spike trains from local field potentials. Journal of Neurophysiology, 99(3), 1461-1476.

Ray, S., Crone, N. E., Niebur, E., Franaszczuk, P. J., \& Hsiao, S. S. (2008). Neural correlates of high-gamma oscillations (60$200 \mathrm{~Hz})$ in macaque local field potentials and their potential implications in electrocorticography. Journal of Neuroscience, 28 (45), 11526-11536.

Ray, S., Hsiao, S. S., Crone, N. E., Franaszczuk, P. J., \& Niebur, E. (2008). Effect of stimulus intensity on the spike-local field potential relationship in the secondary somatosensory cortex. Journal of Neuroscience, 28(29), 7334-7343.

Roopun, A. K., Kramer, M. A., Carracedo, L. M., Kaiser, M., Davies, C. H., Traub, R. D., et al. (2008). Temporal interactions between cortical rhythms. Front Neuroscience, 2(2), 145-154.

Schroeder, C. E., \& Lakatos, P. (2009). Low-frequency neuronal oscillations as instruments of sensory selection. Trends in Neurosciences, 32(1), 9-18.

Schroeder, C. E., Lakatos, P., Kajikawa, Y., Partan, S., \& Puce, A. (2008). Neuronal oscillations and visual amplification of speech. Trends in Cognitive Sciences, 12(3), 106-113.

Shannon, C. E. (1948). A mathematical theory of communication. AT\&T Technical Journal, 27, 379-423.

Siegel, M., \& Konig, P. (2003). A functional gamma-band defined by stimulus-dependent synchronization in area 18 of awake behaving cats. Journal in Neuroscience, 23(10), 4251-4260.

Singh, N. C., \& Theunissen, F. E. (2003). Modulation spectra of natural sounds and ethological theories of auditory processing. Journal of the Acoustical Society of America, 114(6 Pt 1), 3394 3411.

Steriade, M. (2006). Grouping of brain rhythms in corticothalamic systems. Neuroscience, 137(4), 1087-1106.

Strong, S. P., Koberle, R., de Ruyter van Steveninck, R. R., \& Bialek, W. (1998). Entropy and information in neural spike trains. Physical Review Letters, 80(1), 197-200.

Womelsdorf, T., \& Fries, P. (2007). The role of neuronal synchronization in selective attention. Current Opinion in Neurobiology, 17(2), 154-160.

Womelsdorf, T., Fries, P., Mitra, P. P., \& Desimone, R. (2006). Gamma-band synchronization in visual cortex predicts speed of change detection. Nature, 439(7077), 733-736. 